\title{
1 Molecular architecture of black widow spider neurotoxins
}

3 Minghao Chen ${ }^{1,2}$, Daniel Blum ${ }^{3}$, Lena Engelhard ${ }^{2}$, Stefan Raunser $^{2}$, Richard Wagner $^{3}$,

4 Christos Gatsogiannis ${ }^{1,2 *}$

5

6

7 Affiliations

8

$9 \quad{ }^{1}$ Institute for Medical Physics and Biophysics and Center for Soft Nanoscience, Westfälische

10 Wilhelms Universität Münster, 48149 Münster, Germany.

11

$12{ }^{2}$ Department of Structural Biochemistry, Max Planck Institute of Molecular Physiology,

1344227 Dortmund, Germany.

14

15

${ }^{3}$ MOLIFE Research Center, Jacobs University Bremen, 28759 Bremen, Germany.

16

17 *To whom correspondence should be addressed.

18 E-mail: christos.gatsogiannis@uni-muenster.de.

19 


\section{Abstract}

Latrotoxins (LaTXs) are presynaptic pore-forming neurotoxins found in the venom of Latrodectus spiders. The venom contains a toxic cocktail of seven LaTXs, with one of them targeting vertebrates $(\alpha$-latrotoxin $(\alpha$-LTX)), five specialized on insects $(\alpha, \beta, \gamma, \delta, \varepsilon$ latroinsectotoxins (LITs), and one on crustaceans ( $\alpha$-latrocrustatoxin ( $\alpha$-LCT)). LaTXs bind to specific receptors on the surface of neuronal cells, inducing the release of neurotransmitters either by directly stimulating exocytosis or by forming $\mathrm{Ca}^{2+}$-conductive tetrameric pores in the membrane. Despite extensive studies in the past decades, a high-resolution structure of a LaTX is not yet available and the precise mechanism of LaTX action remains unclear.

Here, we report cryoEM structures of the $\alpha$-LCT monomer and the $\delta$-LIT dimer. The structures reveal that LaTXs are organized in four domains. A C-terminal domain of ankyrin-like repeats shields a central membrane insertion domain of six parallel $\alpha$-helices. Both domains are flexibly linked via an $\mathrm{N}$-terminal $\alpha$-helical domain and a small $\beta$-sheet domain. A comparison between the structures suggests that oligomerization involves major conformational changes in LaTXs with longer C-terminal domains. Based on our data we propose a cyclic mechanism of oligomerization, taking place prior membrane insertion. Both recombinant $\alpha$-LCT and $\delta$-LIT

41 form channels in artificial membrane bilayers, that are stabilized by $\mathrm{Ca}^{2+}$ ions and allow calcium 42 flux at negative membrane potentials. Our comparative analysis between $\alpha$-LCT and $\delta$-LIT 43 provides first crucial insights towards understanding the molecular mechanism of the LaTX 44 family. 


\section{Introduction}

Latrotoxins (LaTXs) are potent high molecular weight neurotoxins from the venom of black widow spiders. The venom contains an arsenal of phylum-specific toxins, including one vertebrate-specific toxin, $\alpha$-latrotoxin $(\alpha-\mathrm{LTX})^{1}$, five highly specific insecticidal toxins $(\alpha-, \beta-$, $\gamma-, \delta$-, and $\varepsilon$-latroinsectotoxin (LITs) $)^{2,3}$, and one crustacean-specific toxin, $\alpha$-latrocrustatoxin $(\alpha-\mathrm{LCT})^{3,4}$. The vertebrate specific $\alpha$-LTX causes a clinical syndrome named lactrodectism upon a venomous bite to humans, which is fortunately rarely life-threatening but often characterized by severe muscle cramps and numerous other side effects such as hypertension, sweating, and vomiting ${ }^{5,6}$.

LaTXs are produced as $\sim 160 \mathrm{kDa}$ inactive precursor polypeptides in venom glands and secreted into the gland lumen. There the final mature $130 \mathrm{kDa}$ toxin is produced by proteolytic processing at two furin sites and cleavage of a $\mathrm{N}$-terminal signal peptide and a $\mathrm{C}$-terminal inhibitory domain ${ }^{7,8}$. Most of the physiological and molecular biological researches to date have been carried out using the vertebrate specific toxin $\alpha$-LTX.

$\alpha$-LTXs have been shown to form cation-selective pores upon binding to specific receptors on the presynaptic membrane and induce $\mathrm{Ca}^{2+}$ influx, mimicking thereby physiological voltagedependent calcium channels ${ }^{9,10} . \mathrm{Ca}^{2+}$ influx activates the exocytosis machinery ${ }^{11}$ and triggers massive release of neurotransmitters. $\alpha$-LTX was shown to form also pores on artificial lipid bilayers, which have high conductance for monovalent and divalent cations such as $\mathrm{K}^{+}, \mathrm{Na}^{+}$, $\mathrm{Ca}^{2+}$, and $\mathrm{Mg}^{2+}$, but are blocked by transition metals and trivalent ions such as $\mathrm{Cd}^{2+}$ and $\mathrm{La}^{3+}$ 12-16. Efficient incorporation into biological membranes, strictly relies however on the presence of specific receptors ${ }^{17-19}$.

To date, three receptors for $\alpha$-LTX have been isolated, i.e., the cell adhesion protein neurexin (NRX) which binds to the latrotoxin in a $\mathrm{Ca}^{2+}$-dependent manner ${ }^{20-22}$, the $\mathrm{G}$ protein-coupled receptor latrophilin (LPHN or CIRL, stands for Calcium-Independent Receptor of 
Latrotoxin $^{23,24}$ and the receptor-like protein tyrosine phosphatase $\sigma(\mathrm{PTP} \sigma)^{25}$. NRX and PTP $\sigma$ are suggested to provide with regard to $\alpha$-LTX only a platform for binding and subsequent pore formation events ${ }^{25-29}$. Intriguingly, $\mathrm{Ca}^{2+}$-independent binding to receptor LPHN does not involve in contrast oligomerization and channel formation, but direct downstream stimulation of the synaptic fusion machinery ${ }^{27,30-32}$.

The channel dependent and independent functions of $\alpha$-LTX have attracted the attention of neurobiologists for several decades, studying the effects of $\alpha$-LTX on neurotransmitter release and mechanisms underlying synaptic plasticity. The $\alpha$-LTX variant LTX $^{\mathrm{N} 4 \mathrm{C}} 33$, which lacks the ability of pore-formation but retains the full binding affinity to receptors, played a key role in the investigations of $\alpha$-LTX action. Today, $\alpha$-LTX is an indispensable tool for stimulating exocytosis of nerve and endocrine cells ${ }^{29,34-36}$. $\alpha$-LTXs are furthermore considered to antagonize botulinum poisoning and attenuate the neuromuscular paralysis via synapse remodeling ${ }^{37}$. The surprising structural homology of $\alpha$-LTX to the glucogen-like peptide- 1 (GLP1) receptor might also open opportunities for pharmacological applications in blood glucose normalization and reversion of neuropathies ${ }^{38}$.

Invertebrate LaTXs are less well understood, but considered as promising candidates for the development of novel bio-pesticides. Orthologues of the three receptor classes shown to bind $\alpha$-LTX are also present in insects ${ }^{39}$. To date, four LaTXs have been cloned, including $\alpha$-LTX ${ }^{7}$, $\alpha-\mathrm{LIT}^{40}, \delta$-LIT ${ }^{41}$, and $\alpha-\mathrm{LCT}^{42}$. Despite their high specificity, the different LaTXs display a 30$60 \%$ sequence identity and are expected to share an overall similar domain organization and membrane insertion mechanism. Low resolution 3D maps (14-18 $\AA$ ) of the $\alpha$-LTX dimer, $\alpha$ LTX tetramer, and $\delta$-LIT monomer were previously determined using single particle negative stain and cryoEM ${ }^{39,43}$, suggesting indeed an overall similar architecture of the different members of the LaTX family. 
94 A structural and mechanistic understanding of LaTX function is a significant priority for the

95 development of novel anti-toxin therapeutics and/or insecticides. However, a high-resolution 96 structure of a LaTX, which is a prerequisite for the understanding of LaTXs' mechanism of

97 action at molecular detail, has been missing. Here we present a $4.0 \AA$ cryoEM structure of the $98 \alpha$-LCT monomer and a $4.6 \AA$ cryoEM structure of the $\delta$-LIT dimer revealing the molecular 99 architecture of LaTX neurotoxins as well as the molecular details of their oligomerization

100 mechanism prior to membrane insertion. In addition we characterized the principal basic pore

101 characteristics of $\alpha$-LCT, the precursor $\delta$-LIT and $\delta$-LIT channels after reconstitution into 102 planar lipid bilayer. 


\section{Results}

104 CryoEM structure determination of $\boldsymbol{\alpha}$-LCT

105 We recombinantly expressed the mature $\alpha$-LCT (amino acids 16-1240) (Fig. 1a and

106 Supplementary Fig. 1) in insect cells using the MultiBac system and purified it using a

107 combination of affinity and size exclusion chromatography to obtain a monodisperse sample

108 for cryoEM single particle analysis (Supplementary Fig. 2a, b). The cryoEM sample showed a

109 homogeneous set of characteristic G-shaped flat particles, corresponding to soluble monomers

110 of the $130 \mathrm{kDa}$ mature $\alpha$-LCT complex (prepore state; before membrane insertion). The G-

111 shaped particle is composed of a C-like curved region, corresponding to the long C-terminal

112 domain of ankyrin-like repeats (ARs), that is engulfing a central compact "head" region (Fig.

113 1b, Supplementary Fig. 2c).

114 Subsequent image processing and 3D classifications revealed an inherent flexibility between

115 both regions. The N-terminal head is orientated perpendicular and in close vicinity to the tail

116 of the AR region in the best resolved class ("compact" conformation), but shows a continuous

117 movement and is tilted away the tail of the AR-domain in less well resolved classes, resulting

118 in less compact conformations (Supplementary Fig. 2d,e, 3a).

119 The $\alpha$-LCT monomer in the "compact" conformation adopts a flat architecture and is $130 \AA$

120 long and $30 \AA$ wide. The path of the polypeptide is clear in the density map, allowing us to

121 build an atomic model covering $85 \%$ of the sequence of the molecule (residues 48-1066, except

122 two disordered loop regions 226-232, 349-360) (Supplementary Fig. 4a). We deleted side-chain

123 atoms beyond $\mathrm{C} \beta$ in regions where side-chain density was only rarely evident. The C-terminal

124 end of the ARs domain including the last four ankyrin repeats is not resolved. The local

125 resolution is highest in the N-terminal head region (Supplementary Fig. 2h). 


\section{Architecture of the soluble $\alpha$-LCT monomer}

129 The resulting atomic model reveals that the N-terminal head region is composed of three

130 domains: a four-helix domain at the N-terminal end, termed here as "connector" domain

131 (residues 48-115); a central helical bundle domain (residues 116-352), and a short $\beta$-sheet

132 domain, linking the helical bundle domain with the AR domain, termed here as "plug" domain

133 (residues 353-452) (Fig. 1a,c, Supplementary Fig. 5, Movie 1).

134 The helical bundle domain shows a novel fold of a six-helix bundle, with five parallel aligned

135 helices assembling into a cylindrical structure (H5-7, H9-10), encircling a central $\alpha$-helix (H8)

136 (Fig. 1d). The conserved helix H8 contains many hydrophobic residues and is predicted to act

137 as transmembrane region of the tetrameric LaTX pore (Supplementary Fig. 6d). The

138 surrounding helices shield the hydrophobic surface of $\mathrm{H} 8$ within the cylindrical bundle and

139 protect it from the aqueous environment (Supplementary Fig. 7a,b). The helical bundle domain

140 is expected to undergo severe conformational changes during pore formation to allow exposure

141 of the transmembrane helix $\mathrm{H} 8$ and transition of the toxin from a soluble monomer to a

142 transmembrane tetramer. Interestingly, helices $\mathrm{H} 7$ and $\mathrm{H} 9$ are kinked and interrupted by two

143 (residues 191-196, 203-211) and one (residues 296-304) short loops, respectively. Such short

144 breaks interrupting long $\alpha$-helices in close vicinity to the putative transmembrane regions, were

145 shown in other $\alpha$-helical pore forming toxins to provide the necessary flexibility for major

146 conformational changes towards membrane insertion ${ }^{44}$.

147 The long and curved C-shaped C-terminal AR domain consists of 22 ankyrin-like repeats,

148 accounting for two thirds of the sequence of $\alpha$-LCT. In total, the first 18 out of 22 ankyrin-like

149 repeats (ARs) were resolved in the present map. Interestingly, there is a redirection of

150 orientation of the ARs at the loop (residues 890-902) connecting AR13 and AR14. ARs14-18

151 are rotated almost 90 degrees compared to ARs1-13 along the long axis of the domain (Fig. 1f).

152 This arrangement goes along with a characteristic bipolar charge distribution, with ARs1-13 
153 dominantly positively charged and the tail of ARs14-18 displaying a prominent negatively

154 charged patch (Fig. 1g, Supplementary Fig. 6a).

155 The "connector" domain at the N-terminal end forms the "lower" interface connecting the

156 central helical bundle with the AR-domain (Fig. 1c,e). Helices H1, H2, H4 and the short helix

$157 \mathrm{H} 3$ of the "connector "domain, assemble into a flat triangle structure, which is attached to the

158 inner curved surface of the AR-domain and interacts with ARs 6-10. Most interaction surface

159 to AR-domain is provided by $\mathrm{H} 2$, whereas the short helix $\mathrm{H} 3$ is directly positioned in close

160 proximity to the loop connecting AR9 and AR10 and parallel aligned to the helices of AR9 and

161 AR10 (Fig. 1e). The residues involved in this interface are only conserved in the AR-, not in

162 the "connector" domain (Supplementary Fig. 7c,d). They are mainly hydrophobic, suggesting

163 a major role of hydrophobic interactions. Helix H2 of the "connector" domain is hydrophobic

164 in our structure and interestingly, this helix has been also predicted as the second transmembrane region of the insecticidal $\delta$-LIT (Supplementary Fig. 6h), but not predicted as such from the sequences of other latrotoxin family members, such as $\alpha$-LCT (Supplementary Fig. 6d), or $\alpha$-LTX (data not shown).

168 The "plug"-domain covalently links the primary sequence of the helical bundle with the AR169 domain, positioning the helical bundle directly below AR1 ("upper interface") (Fig. 1c, 170 Supplementary Fig. 7e-g). The "plug"-domain is organized in two layers: a region of several

171 flexible loops and a core region of four $\beta$-strands that is attached to $\mathrm{H} 5$ and a short loop between

$172 \mathrm{H} 8$ and H9 of the helical bundle (Supplementary Fig. 7h). The "plug"-domain plays an 173 important role in the oligomerization of the complex prior complex formation, which will be 174 discussed in detail in the next section.

\section{CryoEM structure of inactive precursor soluble $\boldsymbol{\delta}$-LIT dimer}

177 In subsequent experiments, we were not able to induce oligomerization of $\alpha$-LCT and trigger 178 insertion into liposomes for further visualization of pore formation events, as previously 
described for $\alpha-\mathrm{LTX}^{43,45}$. To provide further insights into the LaTX family, we then focused on

180 the insecticidal $\delta$-LIT. As expected, mature $\delta$-LIT was toxic for our insect cell cultures and

181 therefore, we expressed, purified, and subjected to cryoEM analysis the precursor uncleaved

182 inactive toxin. The precursor toxin contains an additional signal peptide (residues 1-28) and an

183 inhibitory domain ( $\alpha$-LCT residues 1037-1214), compared to the matured form (Fig. 2a). Albeit

184 we performed the cryoEM analysis with the same procedure used for mature $\alpha$-LCT, subsequent

185 processing did not only reveal G-shaped monomers, as was the case for mature $\alpha$-LCT, but also

186 higher order oligomers. In particular, reference free 2D classifications revealed $40 \%$ monomers, 50\% dimers, 5\% trimers and 5\% tetramers (Fig. 2b, Supplementary Fig 8a-c). Interestingly, such particle populations of oligomers were not observed in negative-stain EM (Supplementary

189 Fig. 9a,b), indicating that the interactions are rather weak and dilution of the sample which is

190 necessary for negative-stain EM, as well as the low $\mathrm{pH}$ of the stain might induce dissociation

191 of the oligomers. We were finally able to obtain a map of the $\delta$-LIT dimer at $4.6 \AA$ average resolution from 81,192 particles, with no symmetry imposed (Supplementary Fig. 8d-f).

193 A 3D reconstruction of the $\delta$-LIT monomer was unfortunately not possible, due to preferred 194 orientation of the single particles. Based on the structure of $\alpha$-LCT monomer, we were able to 195 build a molecular model of the $\delta$-LIT dimer, including residues 50-928 for both protomers (Fig. 2c, Supplementary Fig. 4b, Movie 2). As expected, the structure of the protomer of $\delta$-LIT is very similar to that of $\alpha$-LCT (sequence identity 39\%), showing the characteristic G-shaped architecture and domain organization. $\delta$-LIT displays however a shorter repetitive C-terminal AR-domain, containing only 15 ARs instead of 22 in $\alpha$-LCT.

200 The path of the polypeptide is clear for all four domains, i.e., connector domain, helical bundle 201 domain, plug domain, and AR-domain, except for a few loop regions (residues 91-92, 99-105, $237-245,355-365)$ in protomer $A$ and $(237-244,356-361)$ in protomer $B$, the last $\left(15^{\text {th }}\right)$ AR and

203 the complete signal peptide and inhibitory domain in both protomers (Fig. 2c). These structural 204 regions were not resolved in the cryoEM density map, probably because they are disordered or 
highly flexible. Due to limited resolution, we deleted most of the side-chain atoms beyond C $\beta$ in the molecular model unless the electron density was sufficiently clear with regard to bulky side chains.

The $\delta$-LIT dimer is formed with the two protomers rotated 90 degrees relative to each other and the "plug" domain of protomer A plugging from the side into a cleft formed by ARs 1-6 of protomer B (Fig. 2c). The protomers A and B are in basically the same conformation, but the 211 small differences, mainly due to the flexibility of the helical bundle domain, precluded successful C2 symmetrization of the particle (Supplementary Fig. 3e).

The "plug"-domain has a hemispherical architecture matching the cleft formed by the $1^{\text {st }}-6^{\text {th }}$ ARs of the AR-domain (Fig. 2d), suggesting an induced fit and shape complementarity as the basis for the interaction. We found clusters of 17 polar and charged residues on the "plug"domain of protomer A and 18 polar and charged residues on the cleft of protomer B, that may be involved in this interface (Figure 2e, Supplementary Table 1). In previous studies, the $\alpha$ LTX variant $\mathrm{LTX}^{\mathrm{N} 4 \mathrm{C}}$ with an insertion of four amino acids (VPRG) at the linker peptide connecting the plug domain with the AR-domain ${ }^{33}$, was shown to retain its binding affinity to receptors, but lose its ability to oligomerize into tetramers and form pore ${ }^{27}$. This variant played a key role in understanding of the dual mode of action of LaTXs. The corresponding position of this insertion is highlighted on the molecular model of $\delta$-LIT (Fig. 2d). This insertion is not positioned directly at the dimerization interface, but in close proximity to the loops of "plug"domain involved in the interaction and might thus disturb the overall shape complementarity and/or induce a shift of positions and a mismatch between the residues involved in this interface, blocking thereby dimerization.

Besides the main interaction between the "plug"-domain of protomer A and ARs 1-6 of protomer $\mathrm{B}$, there is a less pronounced interaction between the helical bundle domains of both protomers, involving $\mathrm{H} 9$ of protomer A and H6/H7a of protomer B (Fig. 2e, Supplementary 
231 loop in its middle, in protomer B (thus most probably upon dimer formation), this loop folds 232 helically to straighten and complete H9 (Supplementary Fig. 3f, Supplementary Fig. 4b).

233 Interestingly, because $\delta$-LIT is lacking seven terminal ARs, the surface of the AR-domain does 234 not display a clear bipolar charge distribution as in $\alpha$-LCT (Fig. 2f, Supplementary Fig. 6e). 235 However, in both LaTXs, the terminal tail of the AR-domain is clearly negatively charged 236 (Supplementary Fig. 6a, e).

Structural differences between $\delta$-LIT and $\alpha$-LCT

239 In comparison to the "compact" conformation of the $\alpha$-LCT monomer, both protomers of the $\delta$-LIT dimer show a different, rather "extended" conformation: the outermost helix H9 and the overall helical bundle domain are straightened, the helical bundle of each protomer is further 242 tilted 15 degrees away the long axis of the AR-domain and the distance between the helical bundle domain and the shorter AR-domain is substantially longer. Notably, the AR-domain of $\delta$-LIT is significantly less curved (Fig. 3a) and its C-terminal tail is further 'twisted' and positioned outside the helical bundle domain and ankyrin-like repeat domain (HBD-ARD) plane (Fig. 3b). As a consequence, the bottom part of the helical bundle domain becomes in this case exposed (Fig. 3c).

The enlarged distance between AR-domain and helical bundle domain, requires also a more extended conformation of the connector domain, which is further stretched in $\delta$-LIT, but still bridges both domains. This results into repositioning of $\mathrm{H} 1$ and unfolding of the lower half of H4 of the connector domain (Fig. 3e). These differences between the compact $\alpha$-LCT and the extended $\delta$-LIT are summarized in Movie 3 and a schematic diagram in Fig. 3f. Interestingly, the less well resolved 3D class of $\alpha$-LCT can be considered as flexible intermediate between both structures (Supplementary Fig 3g, h, Movie3).

We were not able to obtain a structure of the $\delta$-LIT monomer, but the respective 2D class averages, suggest also flexibility for the helical bundle domain and possibly a mixture of 
different compact, intermediate and extended conformations (Fig. 2b). $\alpha$-LCT appears in general however more compact, due to the seven additional terminal ARs, allowing additional interactions between the AR-domain and the flexible central helical bundle. This might explain

conditions. The directional change observed however in the AR-domain of the "extended" $\delta$ -

LIT protomer, would also result in exposure of the helical bundle domains, even for LaTXs

264 It should be noticed that the previous low resolution EM studies on $\alpha$-LTX and $\delta$-LIT show an the present 2D classes of $\delta$-LIT dimers and tetramers (Fig. 2b) display however clear similarities, indicating structure conservation. Taking in addition in account the high sequence

268 similarity within the LaTX family, we rather conclude that the earlier LaTX reconstructions determined more than two decades ago, are apparently affected by the previous bottlenecks of the technique and the significantly lower signal to noise ratio in the cryoEM micrographs. The members of the LaTX family share an overall common domain organization and architecture.

\section{Electrophysiological characteristics of the pore forming precursor $\delta$-LIT in comparison} with the mature $\delta$-LIT and $\alpha$-LCT

275 We further performed electrophysiology studies to demonstrate pore-formation activity of the 276 recombinant proteins and provide a detailed characterization for the less well studied 277 invertebrate LaTX channels. One important aspect herein is the role of the C-terminal domain 278 in channel formation, since its cleavage is required for activation of the toxins. Therefore, we 279 additionally prepared precursor full length $\delta$-LIT for further functional comparisons with 280 mature truncated $\delta$-LIT and $\alpha$-LCT. The inherent cleavage sites were however not recognized 281 by furin protease (data not shown) and therefore we inserted two additional cleavage sites into 282 the sequence of precursor $\delta$-LIT, before the C-terminal- (residue 29) and after the AR-domain 
(residue 1019) followed by proteolysis treatment after expression (Supplementary Fig. 9c,d).

284 The two mature toxins and precursor $\delta$-LIT were then reconstituted into planar lipid bilayer and 285 voltage depended membrane currents $\left(V_{\text {mem }}\right.$, membrane potential) were recorded at single 286 channel resolution as previously described ${ }^{46}$.

287 Precursor $\delta$-LIT spontaneously inserted into the lipid bilayer and formed open channels as 288 obvious from the observed large voltage induced currents (Supplementary Fig. 10a,c). With asymmetric (cis/trans) $150 / 25 \mathrm{mM} \mathrm{KCl}$ buffer conditions, the reversal potential $\left(V_{\text {rev }}\right)$ was $V_{\text {rev }}=4 \pm 1 \mathrm{mV}$ yielding $P_{K^{+}} / P_{C l^{-}}=1.25^{47,48}$, demonstrating only marginal selectivity to $\mathrm{K}^{+}$over $\mathrm{Cl}^{-}$ions (Fig. 4a). However, upon addition of $10 / 1 \mathrm{mM} \mathrm{CaCl}_{2}$ (cis/trans)) gradient in symmetrical $150 / 150 \mathrm{mM} \mathrm{KCl}$ buffer, we observed an approximate five-fold increase in the reversal potential $\left(V_{\text {rev }}=20 \pm 2,5 \mathrm{mV}\right.$ (Fig. 4b)) revealing that the precursor $\delta$-LIT voltage activated channel preferentially conducts $\mathrm{Ca}^{2+}$ ions $\left(P_{\mathrm{Ca}^{2+}} / P_{C l^{-}}=18\right)$ and the current-voltage relation became rectifying. Moreover, calcium stabilized the channel, visible by a significant reduction in the current noise. The mean main conductance as determined from all point current histograms at different voltages was $\bar{G}_{\text {main }}=330 \pm 36 p S(N=12)$

The current voltage relations of membrane inserted mature $\delta$-LIT in symmetrical 250/250 mM

$\mathrm{KCl}$ buffer showed a linear course with high gating activity(Fig. 4c). In contrast to the precursor

300 variant, we observed low noise currents with structured gating which could be resolved in amplitude and time. Similarly to the precursor-, mature $\delta$-LIT channels display also only a slight cation selectivity $\left(P_{K^{+}} / P_{C l^{-}}=1.47\right.$, Fig. 4 d) after establishing the $250 / 25 \mathrm{mM} \mathrm{KCl}$ gradient. Addition of $5 \mathrm{mM} \mathrm{Ca}^{2+}$ ions to the cis side of the bilayer changed however the 304 properties of the mature $\delta$-LIT channel completely. The asymptotic-sine course of the current305 voltage relation increased at both negative and positive command voltages $\left(V_{c m d}\right)$ while crossing the zero voltage with zero net current (Fig. 4f). To further analyze this, we applied the 307 widely used Goldman-Hodgkin-Katz (GHK) approach ${ }^{47,48} 49$ (see Supplemental for details). It 
turned out that the course of the current-voltage relation in Figure 4f can be explained if the currents at negative $V_{c m d}$ are carried from cis to trans mainly by $\mathrm{Ca}^{2+}$-ions and at positive $V_{c m d}$ predominantly by $\mathrm{Cl}^{-}$ions (Fig. 4e; see Supplemental for details). The analysis of current traces revealed two open channel states with mean amplitudes of $\bar{G}_{1}=25 \pm 2.8 p S$ and $\bar{G}_{2}=180 \pm$ $24.8 \mathrm{pS}$ corresponding to a pore restriction diameter of about $0.8 \mathrm{~nm}^{48,50}$ (Supplementary Fig. $10 \mathrm{e}, \mathrm{f})(\mathrm{N}=15)$ significantly smaller than the one calculated for the precursor $\delta$-LIT channel

$314(\sim 1.5 \mathrm{~nm}$ see Supplementary Figure 10c). Thus the mature $\delta$-LIT channel appears to form a denser, stabilized conformation compared to its precursor variant. Additionally, the mature $\delta$ LIT seems to act like a complete rectifier, which in the presence of $\mathrm{Ca}^{2+}$, depending on membrane polarization, allows mainly either flux of calcium $\left(-V_{m e m}\right)$ or chloride currents the mature $\delta$-LIT are incorporated into the bilayer predominantly in a unidirectional manner, with a regulative $\mathrm{Ca}^{2+}$-binding site on the cis side. Strong rectifying current-voltage relations were observed in all bilayer experiments in the presence of $\mathrm{Ca}^{2+}$-ions added on the cis side

Surprisingly, mature $\alpha$-LCT incorporates into the bilayer membrane and forms channels only in the presence of $\mathrm{Ca}^{2+}$-ions (cis) (Supplementary Fig 10g). The analysis of the single channel traces (Supplementary Fig 10h,i) revealed, similar to mature $\delta$-LIT, two distinct open channel states $\bar{G}_{1}=78 \pm 5.6 p S$ and $\bar{G}_{2}=180 \pm 17.3 p S(\mathrm{~N}=7)$ (Supplementary Fig 10i). Beside the $10 / 1 \mathrm{mM} \mathrm{CaCl}_{2}$ gradient, the $\alpha$-LCT channel does not show, in contrast to $\delta$-LIT, any preference 328 for the involved anions and cations ( $V_{\text {rev }}=0 \pm 2.1 \mathrm{mV}$ (Supplementary Fig. $10 \mathrm{~g}$ ). In comparison to mature $\delta$-LIT (Fig. 4f), the $\alpha$-LCT channel displays a higher current noise level

330 in the recordings and surprisingly, considering the slightly asymptotic sine course of the 331 current-voltage relation (Supplementary Fig 10g), the $\alpha$-LCT apparently also harbors rectifying 
332 properties, making it likely that rectified currents of $\mathrm{Ca}^{2+}$-ion and $\mathrm{Cl}^{-}$-ions may convey 333 similar to mature $\delta$-LIT.

334 To sum up, $\mathrm{Ca}^{2+}$-ions appear to further stabilize precursor and mature LaTX oligomers after

335 incorporation into the membrane resulting in a rectifying calcium selective channel allowing

336 calcium flux at negative membrane potentials. High $\mathrm{Ca}^{2+}$ permeability was previously also 337 shown for native $\delta$-LIT channels in locust muscle membrane and artificial bilayer, but not for 338 truncated recombinant variants ${ }^{41}$. Provided that as a result of the predominantly unidirectional 339 insertion the cis side corresponds to the cytosolic side of the respective channel, $\delta$-LIT and $\alpha$ 340 LCT display ion channel characteristics similar to $\mathrm{Ca}^{2+}$-release channels ${ }^{51}$.

\section{Formation of an insertion-competent prepore complex}

343 The reference free 2D class averages of monomers, dimers, trimers and tetramers identified in

344 the $\delta$-LIT cryoEM dataset (Fig. 2b) suggest a general sequential oligomerization mechanism of

345 LaTXs towards the formation of a soluble tetrameric complex prior membrane insertion. The

346 bent cleft at the upper part of the AR-domain of a protomer is employed as a binding site for

347 the hemispherical plug domain of an adjacent protomer, with both molecules rotated 90 degrees

348 relative to each other. Four LaTX molecules dock into each other in a sequential circular ring-

349 fashion form via 1/2/3-mers to eventually form tetramers (Supplementary Fig. 11a). This is to

350 our knowledge the first description of a LaTX intermediate trimer, suggesting that the tetramer

351 is not exclusively formed upon assembly of two dimers, as previously proposed ${ }^{45}$.

352 Based on our data, we generated a 3D model of the tetramer by arranging the $\delta$-LIT dimer

353 ("extended state") according the 2D class averages of the top view of the tetramer. The resulting

354 3D model of the tetramer is approximately $140 \times 140 \AA$ A large and has a height of approximately

$355120 \AA$, with an overall striking configuration resembling a "four-finger crane claw", with each curved AR-domain resembling a finger of the crane claw (Fig. 5a). 
In the resulting model, the four cylindrical helical bundle domains are surrounding a central 10

$358 \AA$ diameter channel, which agrees excellent with the 2D class averages of the soluble $\delta$-LIT tetramer (Fig. 2b) and the previous 2D class averages of the soluble $\alpha$-LTX tetramer ${ }^{43}$. The putative transmembrane helices are still however completely shielded from the aqueous environment within the respective helical bundle domains. Nevertheless, the bottom part of the

362 helical bundle domains is exposed in this arrangement. We therefore propose that the tetramer,

363 shown here, composed of "extended" protomers, resembles an insertion-competent prepore state of the toxin.

Assembling the tetramer in a similar manner from $\alpha$-LCT "compact" protomers with 22 ARs results instead into a completely closed "crane claw" (Supplementary Fig. 11b). In this scenario, the AR-domains shield the central helical bundle domains from both sides and the central channel is closed. The resulting compact structure does not match however the 2D class averages of LaTX tetramers (Fig. 2b and Orlova et. al. ${ }^{43}$ ) and there are severe clashes between the AR-domains of adjacent subunits. Note that in previous 2D class averages of $\alpha$-LTX tetramers with $22 \mathrm{ARs}^{43}$, the central helical bundle domains are also exposed, suggesting that 372 the single protomers are in the "extended" conformation. This suggests that $\alpha$-LCT has to 373 undergo a conformational switch from the compact to extended conformation during the oligomerization process and not after formation of the tetramer. Low resolution 3D classes of

375 the $\alpha$-LCT monomer resembling intermediate states between the compact and the extended conformation further support this scenario (Supplementary Fig. 3g,h, Supplementary Movie 3). $\alpha$-LCT shows an intriguing bipolar charge contribution, which is apparently present also in $\alpha$ 378 LTX which has also 22 ARs, but however less pronounced in $\delta$-LIT with 15 ARs 379 (Supplementary Fig. 12, Supplementary Fig. 6a,e). The "claw tips" (lower ends of the ARdomains) are however clearly negatively charged in all LaTXs. 


\section{Discussion}

\section{Oligomerization characteristics and function of the inhibitory domain}

385 Albeit extensive efforts, also in presence of artificial membranes, we were not able to determine in vitro, in absence of receptors, factors controlling the oligomerization and subsequent pore formation process efficiently for further visualization of the pore formation events. Interestingly, we observed the various populations of prepore oligomers of precursor $\delta$-LIT $(5 \%$ of the particles) only in cryoEM samples (Supplementary Fig. 8b,c) but not during negative stain EM analysis (Supplementary Fig. 9a,b) or other complementary methods (e.g. SEC-MALS, data not shown). The oligomerization occurred in buffer containing EDTA, and the substitution of EDTA with $\mathrm{Mg}^{2+}$ or $\mathrm{Ca}^{2+}$ did not affect the sample (data not shown). This observation corresponds to a previously reported study ${ }^{45}$, suggesting oligomerization of $\delta$-LIT as a process independent of divalent cations. Considering in addition that active $\alpha$-LCT formed exclusively stable monomers throughout our experiments, as well as previous studies suggesting that $\alpha$ -

396 LTX exists mainly in its dimerized or tetramerized form, we conclude that different LaTXs 397 display different oligomerization characteristics. Indeed, although the dimerization interface 398 suggests a general induced fit mechanism in LaTXs, the surface of the involved AR-domain 399 cleft is not highly conserved in the LaTX family (Supplementary Fig. 1 and Supplementary 400 Fig.6c, g).

401 The electrophysiological analysis at single channel resolution allowed us to confirm the 402 tetramerization process for both recombinant expressed LaTX samples (precursor $\delta$-LIT and 403 mature $\alpha$-LCT) in an indirect way, since a complete LaTX tetramer is the prerequisite of 404 functional pore-formation and thus enabling measurements of single channel currents. Indeed, 405 our samples showed full activity and ion channel gating events of single pore units were 406 detected under the conditions of the high resolution electrophysiological experiment. This is in 
also shown to form pores on artificial lipid bilayers, but efficient incorporation into biological membranes was only achieved in presence of specific receptors ${ }^{17-19}$. With regard however to our experiments on $\delta$-LIT, we assume that rapid concentration of the sample during cryoEM

411 grid preparation might have been an important factor, for successful assembly of the soluble

412 tetramer, even for a small subpopulation of particles. The charge on the artificial bilayers might

413 be another important factor for efficient LaTX recruitment and subsequent pore formation in 414 the electrophysiology experiments, due to the bipolar charge distribution of the AR-domains. 415 Under physiological conditions, the individual LaTX receptors are however apparently the 416 critical factors for efficient toxin recruitment, assembly of the tetramer and subsequent pore 417 formation ${ }^{17-19}$. Similar dependencies are well known for prepore oligomers of other toxins 418 assembling at the membrane prior pore formation ${ }^{52}$.Oligomerization might also be reinforced 419 by additional factors in the venom of the spider or receptor mediated interactions at the outer cell surface. Latrodectins, low molecular weight proteins characterized from the black widow venom, are known for example to associate to LaTXs and suspected to enhance their potency by altering the local ion balance ${ }^{53}$.

\section{Implications for latrotoxin receptor recognition, pore-formation and $\mathrm{Ca}^{2+}$ sensing}

425 Neurexin and latrophilin are two well-studied receptors of latrotoxin ${ }^{36,54}$, but the receptor 426 binding site (also for their respective invertebrate homologues) on LaTXs is still unknown. On 427 the one hand, the helical bundle, connector and plug domains are buried deeply in the "crane 428 claw"-like tetramer and the empty space below the helical bundles is most probably required 429 for subsequent pore formation events (Fig. 5a). The four outer "claw fingers" formed by AR430 domains contribute on the other hand the largest exposed surface for receptor interaction and 431 indeed, ankyrin-like repeat domain are widely involved in maintaining protein-protein 432 interactions, as the large numbers of modular repeats and adaptive surface residues make the 433 motif a versatile protein binding partner ${ }^{55,56}$. In addition, our data suggest, that the inhibitory 
434 domain located at the tail of the AR-domain, probably interrupts the toxin-receptor interface.

435 Therefore, LaTX uses most likely the lower half ARs for receptor recognition. Different LaTXs

436 vary indeed in the number of their ARs, but high resolution structures of LaTX-receptor

437 complexes are now necessary, towards understanding their specificity in detail.

438 Interestingly, the helical bundle domain of LaTX is reminiscent of domain I of the pore forming

439 Cry toxin, in which six (but not five) amphipathic helices surround a hydrophobic central helix.

440 Moreover, an $\alpha$-helix in Cry toxin domain I is also interrupted by a short loop ${ }^{57,58}$ as we

441 observed in the $\mathrm{H} 7$ and $\mathrm{H} 9$ helices in our structure. Although the exact pore formation

442 mechanism of Cry toxins is yet unknown, an 'umbrella' model of toxin-insertion, derived from

443 structural studies on colicin, is widely accepted ${ }^{59}$. Recently, the RhopH complex, a pore

444 forming protein of malaria parasites was in addition shown to possess an intriguing similar

445 helical bundle domain to the helical bundle domain of $\operatorname{LaTX}^{60}$. This suggests a common

446 strategy in until recently unrelated pore forming proteins, to protect central hydrophobic surface

447 helices prior membrane insertion, but further studies are now required to unravel possible

448 similarities in the respective pore formation mechanisms.

449 It is known that LaTX pores are permeable for cations and small molecules such as ATP or

450 acetylcholine. The present electrophysiological measurement further reveal conductance values

451 that in the order of magnitude typically observed for , than $\mathrm{K}^{+}$or $\mathrm{Ca}^{2+}$ specific channels ${ }^{61,62}$.

452 The LaTX pore is stabilized by $\mathrm{Ca}^{2+}$ and has a preferred permeability for this ion, suggesting

453 binding sites in the LaTX pore specialized for $\mathrm{Ca}^{2+}$ sensing. A flexible loop in the pore can

454 match both requirements: four loops might form an ion filter-like structure through coordinate

455 bonds with a $\mathrm{Ca}^{2+}$, which can further facilitate the intake of the following $\mathrm{Ca}^{2+}$. In the absence

456 of $\mathrm{Ca}^{2+}$, the loops become disordered, as observed for KcsA in the absence of $\mathrm{K}^{+}$-ions in the

457 filter region ${ }^{63}$, but the pore might be then large enough to pass through the other substrates.

458 Aspartic acid (Asp) and glutamic acid (Glu) residues have been known to play a critical role in

$459 \mathrm{Ca}^{2+}$ filtering ${ }^{62,64}$. There are three Asp residues and five Glu residues strictly conserved at the 
460 helical bundle domain: Asp232, Asp289, Asp427, Glu121, Glu132, Glu146, Glu185, and

461 Glu203 (residue numbers according to $\delta$-LIT).

\section{LaTX mechanism of oligomerization prior membrane insertion}

Taking all observations together, we propose a four-step mechanism of oligomerization and membrane binding of LaTXs (Figure 5b). Firstly, the inhibitory domain of LaTX is removed by proteolytic cleavage. This enables the toxins ("compact" and flexible intermediate conformations") to be recognized by receptors at the extracellular side of the cell membrane. The negatively charged C-terminal tails of AR-domains are further attracted by the cations (e.g. $\mathrm{Na}^{+}$or $\mathrm{Ca}^{2+}$ ) at the extracellular side of the cell membrane, which might be crucial to orientate the molecules properly, with the "claw tips" directly facing the membrane. Adjacent protomers plug to each other via their AR-domains and plug domains in a cyclic sequential manner towards the formation of the tetramer. For $\alpha$-LCT (and possibly also for other LaTXs with 22 ARs), this interaction is expected in addition to trigger a conformational change for each protomer and stabilize an extended conformation. The resulting tetramer resembles in shape an open "crane claw" (prepore; membrane insertion competent state). Notably, in this orientation, the bottom part of the cylindrical helical bundle domains, composed each of five parallel aligned helices protecting the central putative transmembrane helix, are exposed, perpendicular aligned and directly facing the membrane. This orientation towards the membrane, suggests that subsequent pore formation events, might involve massive rearrangements within the four helical bundle domains, resulting, among other events, into

481 downwards injection of the shielded $\mathrm{H} 8$ helices for synchronized membrane penetration, 


\section{Concluding remarks}

487 Our cryoEM results reveal the general architecture of LaTXs and allow us in combination with 488 first functional studies to understand key steps of LaTX action at molecular level. Future studies 489 of receptor-bound LaTXs in membrane inserted state together with mutational analysis of $\mathrm{Ca}^{2+}$ 490 sensing candidate loops and subsequent electrophysiological studies will be necessary to shed 491 light into the intriguing structure and physiological function of the LaTX pore. 


\section{Acknowledgments}

494 We thank Dr. O. Hofnagel and Dr. D. Prumbaum for assistance with dataset acquisition and the 495 development team of the SPHIRE software suite for assistance with image processing. This 496 work was supported by funds from Uehara Memorial Foundation Overseas Postdoctoral 497 Fellowships, Japan Society for the Promotion of Science (JSPS) Overseas Research 498 Fellowships and the Humboldt Research Fellowship for Postdoctoral Researchers (to M.C.), 499 the Max Planck Society (to S.R.) and the Medical Faculty of the University of Münster (to 500 C.G.).

501

\section{Author Contributions}

503 C.G. conceived and supervised the study, M.C. designed latrotoxin expression and purification 504 experiments, M.C., L.E. purified samples, performed cryoEM experiments and processed

505

506

507

508 cryoEM data; M.C. analyzed cryoEM data, performed model building and interpreted data with contributions from S.R. and C.G.; D.B. performed electrophysiological analysis. R.W., D.B. analyzed electrophysiological data, M.C. drafted the manuscript, C.G., R.W. wrote the manuscript, S.R., R.W., C.G. revised the manuscript. All authors read and approved the final manuscript.

\section{Data Availability}

The cryoEM maps of $\alpha$-LCT monomer and $\delta$-LIT dimer have been deposited to the Electron Microscopy Data Bank (EMDB) under the accession codes XXX and XXX. The respective cryoEM datasets have been deposited to EMPIAR under accession codes $\mathrm{XXX}$ and $\mathrm{XXX}$. The coordinates of the corresponding models have been deposited to the Protein Data Bank (PDB) under accession codes XXX and XXX. Other data are available from the corresponding author upon reasonable request. 
bioRxiv preprint doi: https://doi.org/10.1101/2021.04.19.440504; this version posted April 20, 2021. The copyright holder for this preprint

(which was not certified by peer review) is the author/funder. All rights reserved. No reuse allowed without permission.

518

\section{Competing interests}

520 The authors declare no competing interests. 


\section{References}

5221 Tzeng, M. C. \& Siekevitz, P. The effect of the purified major protein factor (alphalatrotoxin) of black widow spider venom on the release of acetylcholine and norepinephrine from mouse cerebral cortex slices. Brain Res 139, 190-196, doi:10.1016/0006-8993(78)90073-2 (1978).

2 Krasnoperov, V. G., Shamotienko, O. G. \& Grishin, E. V. [Isolation and prope tredecimguttatus]. Bioorg Khim 16, 1138-1140 (1990).

3 Grishin, E. V. Black widow spider toxins: the present and the future. Toxicon 36, 1693-1701, doi:10.1016/s0041-0101(98)00162-7 (1998).

4 Krasnoperov, V. G., Shamotienko, O. G. \& Grishin, E. V. [A crustacean-specific neurotoxin from the venom of the black widow spider Latrodectus mactans tredecimguttatus]. Bioorg Khim 16, 1567-1569 (1990).

5 Muller, G. J. Black and brown widow spider bites in South Africa. A series of 45 cases. S Afr Med J 83, 399-405 (1993).

6 Zukowski, C. W. Black widow spider bite. J Am Board Fam Pract 6, 279-281 (1993).

7 Kiyatkin, N. I., Dulubova, I. E., Chekhovskaya, I. A. \& Grishin, E. V. Cloning and structure of cDNA encoding alpha-latrotoxin from black widow spider venom. FEBS Lett 270, 127-131, doi:10.1016/0014-5793(90)81250-r (1990). expression of alpha-latrotoxin in baculovirus system. FEBS Lett 442, 25-28, doi:10.1016/s0014-5793(98)01624-x (1999). calcium fluxes and transmitter release in a neurosecretory cell line. Nature $\mathbf{2 8 3}, \mathbf{7 7 4 -}$ 776, doi:10.1038/283774a0 (1980).

10 Hurlbut, W. P., Chieregatti, E., Valtorta, F. \& Haimann, C. Alpha-latrotoxin channels in neuroblastoma cells. J Membr Biol 138, 91-102, doi:10.1007/BF00211072 (1994).

11 Rizo, J. Mechanism of neurotransmitter release coming into focus. Protein Sci 27, 1364-1391, doi:10.1002/pro.3445 (2018).

12 Finkelstein, A., Rubin, L. L. \& Tzeng, M. C. Black widow spider venom: effect of purified toxin on lipid bilayer membranes. Science 193, 1009-1011, doi:10.1126/science.948756 (1976).

13 Mironov, S. L., Sokolov Yu, V., Chanturiya, A. N. \& Lishko, V. K. Channels produced by spider venoms in bilayer lipid membrane: mechanisms of ion transport and toxic action. Biochim Biophys Acta 862, 185-198, doi:10.1016/00052736(86)90482-7 (1986).

14 Robello, M., Fresia, M., Maga, L., Grasso, A. \& Ciani, S. Permeation of divalent cations through alpha-latrotoxin channels in lipid bilayers: steady-state current-voltage relationships. J Membr Biol 95, 55-62, doi:10.1007/BF01869630 (1987).

15 Scheer, H. W. Interactions between alpha-latrotoxin and trivalent cations in rat striatal synaptosomal preparations. J Neurochem 52, 1590-1597, doi:10.1111/j.14714159.1989.tb09213.x (1989).

16 Rosenthal, L., Zacchetti, D., Madeddu, L. \& Meldolesi, J. Mode of action of alphalatrotoxin: role of divalent cations in $\mathrm{Ca} 2(+)$-dependent and $\mathrm{Ca} 2(+)$-independent effects mediated by the toxin. Mol Pharmacol 38, 917-923 (1990).

17 Hlubek, M. D., Stuenkel, E. L., Krasnoperov, V. G., Petrenko, A. G. \& Holz, R. W. Calcium-independent receptor for alpha-latrotoxin and neurexin 1alpha [corrected] facilitate toxin-induced channel formation: evidence that channel formation results 
from tethering of toxin to membrane. Mol Pharmacol 57, 519-528, doi:10.1124/mol.57.3.519 (2000).

18 Van Renterghem, C. et al. alpha-latrotoxin forms calcium-permeable membrane pores via interactions with latrophilin or neurexin. Eur J Neurosci 12, 3953-3962, doi:10.1046/j.1460-9568.2000.00282.x (2000).

Volynski, K. E. et al. Latrophilin, neurexin, and their signaling-deficient mutants facilitate alpha -latrotoxin insertion into membranes but are not involved in pore formation. J Biol Chem 275, 41175-41183, doi:10.1074/jbc.M005857200 (2000).

20 Petrenko, A. G. et al. Isolation and properties of the alpha-latrotoxin receptor. EMBO J 9, 2023-2027 (1990).

21 Ushkaryov, Y. A., Petrenko, A. G., Geppert, M. \& Sudhof, T. C. Neurexins: synaptic cell surface proteins related to the alpha-latrotoxin receptor and laminin. Science 257, 50-56, doi:10.1126/science.1621094 (1992).

22 Davletov, B. A., Krasnoperov, V., Hata, Y., Petrenko, A. G. \& Sudhof, T. C. High affinity binding of alpha-latrotoxin to recombinant neurexin I alpha. J Biol Chem 270, 23903-23905, doi:10.1074/jbc.270.41.23903 (1995).

23 Davletov, B. A., Shamotienko, O. G., Lelianova, V. G., Grishin, E. V. \& Ushkaryov, Y. A. Isolation and biochemical characterization of a $\mathrm{Ca} 2+-$ independent alphalatrotoxin-binding protein. J Biol Chem 271, 23239-23245, doi:10.1074/jbc.271.38.23239 (1996).

24 Krasnoperov, V. G. et al. The calcium-independent receptor of alpha-latrotoxin is not a neurexin. Biochem Biophys Res Commun 227, 868-875, doi:10.1006/bbrc. 1996.1598 (1996).

25 Krasnoperov, V. et al. Protein-tyrosine phosphatase-sigma is a novel member of the functional family of alpha-latrotoxin receptors. J Biol Chem 277, 35887-35895, doi:10.1074/jbc.M205478200 (2002).

26 Sugita, S., Khvochtev, M. \& Sudhof, T. C. Neurexins are functional alpha-latrotoxin receptors. Neuron 22, 489-496, doi:10.1016/s0896-6273(00)80704-7 (1999).

27 Volynski, K. E. et al. Mutant alpha-latrotoxin (LTXN4C) does not form pores and causes secretion by receptor stimulation: this action does not require neurexins. J Biol Chem 278, 31058-31066, doi:10.1074/jbc.M210395200 (2003).

28 Lajus, S. \& Lang, J. Splice variant 3, but not 2 of receptor protein-tyrosine phosphatase sigma can mediate stimulation of insulin-secretion by alpha-latrotoxin. $J$ Cell Biochem 98, 1552-1559, doi:10.1002/jcb.20871 (2006).

29 Silva, J. P., Suckling, J. \& Ushkaryov, Y. Penelope's web: using alpha-latrotoxin to untangle the mysteries of exocytosis. J Neurochem 111, 275-290, doi:10.1111/j.14714159.2009.06329.x (2009).

30 Davletov, B. A. et al. Vesicle exocytosis stimulated by alpha-latrotoxin is mediated by latrophilin and requires both external and stored Ca2+. EMBO J 17, 3909-3920, doi:10.1093/emboj/17.14.3909 (1998).

31 Ashton, A. C. et al. alpha-Latrotoxin, acting via two Ca2+-dependent pathways, triggers exocytosis of two pools of synaptic vesicles. J Biol Chem 276, 44695-44703, doi:10.1074/jbc.M108088200 (2001).

32 Capogna, M., Volynski, K. E., Emptage, N. J. \& Ushkaryov, Y. A. The alphalatrotoxin mutant LTXN4C enhances spontaneous and evoked transmitter release in CA3 pyramidal neurons. J Neurosci 23, 4044-4053 (2003).

33 Ichtchenko, K. et al. alpha-latrotoxin action probed with recombinant toxin: receptors recruit alpha-latrotoxin but do not transduce an exocytotic signal. EMBO J 17, 61886199, doi:10.1093/emboj/17.21.6188 (1998).

34 Sudhof, T. C. alpha-Latrotoxin and its receptors: neurexins and CIRL/latrophilins. Annu Rev Neurosci 24, 933-962, doi:10.1146/annurev.neuro.24.1.933 (2001). 
$62035 \quad$ Ushkaryov, Y. Alpha-latrotoxin: from structure to some functions. Toxicon 40, 1-5,

621

622

623

624

625

626

627

628

629

630

631

632

633

634

635

636

637

638

639

640

641

642

643

644

645

646

647

648

649

650

651

652

653

654

655

656

657

658

659

660

661

662

663

664

665

666

667

668 doi:10.1016/s0041-0101(01)00204-5 (2002).

36 Ushkaryov, Y. A., Rohou, A. \& Sugita, S. alpha-Latrotoxin and its receptors. Handb Exp Pharmacol, 171-206, doi:10.1007/978-3-540-74805-2_7 (2008).

37 Mesngon, M. \& McNutt, P. Alpha-latrotoxin rescues SNAP-25 from BoNT/Amediated proteolysis in embryonic stem cell-derived neurons. Toxins (Basel) 3, 489503, doi:10.3390/toxins3050489 (2011).

38 Holz, G. G. \& Habener, J. F. Black widow spider alpha-latrotoxin: a presynaptic neurotoxin that shares structural homology with the glucagon-like peptide-1 family of insulin secretagogic hormones. Comp Biochem Physiol B Biochem Mol Biol 121, 177184, doi:10.1016/s0305-0491(98)10088-3 (1998).

39 Rohou, A., Nield, J. \& Ushkaryov, Y. A. Insecticidal toxins from black widow spider venom. Toxicon 49, 531-549, doi:10.1016/j.toxicon.2006.11.021 (2007).

40 Kiyatkin, N., Dulubova, I. \& Grishin, E. Cloning and structural analysis of alphalatroinsectotoxin cDNA. Abundance of ankyrin-like repeats. Eur J Biochem 213, 121127, doi:10.1111/j.1432-1033.1993.tb17741.x (1993).

41 Dulubova, I. E. et al. Cloning and structure of delta-latroinsectotoxin, a novel insectspecific member of the latrotoxin family: functional expression requires C-terminal truncation. J Biol Chem 271, 7535-7543, doi:10.1074/jbc.271.13.7535 (1996).

42 Volynski, K. E. et al. [Molecular cloning and primary structure of cDNA fragment for alpha-latrocrustatoxin from black widow spider venom]. Bioorg Khim 25, 25-30 (1999).

43 Orlova, E. V. et al. Structure of alpha-latrotoxin oligomers reveals that divalent cation-dependent tetramers form membrane pores. Nat Struct Biol 7, 48-53, doi:10.1038/71247 (2000).

44 Gatsogiannis, C. et al. Membrane insertion of a Tc toxin in near-atomic detail. Nat Struct Mol Biol 23, 884-890, doi:10.1038/nsmb.3281 (2016).

45 Ashton, A. C. et al. Tetramerisation of alpha-latrotoxin by divalent cations is responsible for toxin-induced non-vesicular release and contributes to the $\mathrm{Ca}(2+)$ dependent vesicular exocytosis from synaptosomes. Biochimie 82, 453-468, doi:10.1016/s0300-9084(00)00199-1 (2000).

46 Bartsch, P., Harsman, A. \& Wagner, R. Single channel analysis of membrane proteins in artificial bilayer membranes. Membrane Biogenesis: Methods and Protocols, 345361 (2013).

47 Goldman, E. Potentials impedance, and rectification in membranes. J Gen Physiol 27, 37-60 (1943).

48 Hille, B. Ionic Channels of Excitable Membranes. Vol. 3 (Sinauer Ass. Inc., 2001).

49 Ghai, I. et al. General Method to Determine the Flux of Charged Molecules through Nanopores Applied to $\beta$-Lactamase Inhibitors and OmpF. The Journal of Physical Chemistry Letters 8, 1295-1301 (2017).

50 Smart, O. S., Breed, J., Smith, G. R. \& Sansom, M. S. P. A novel method for structure-based prediction of ion channel conductance properties. Biophysical Journal 72, 1109-1126 (1997).

51 Santulli, G., Nakashima, R., Yuan, Q. \& Marks, A. R. Intracellular calcium release channels: an update. J Physiol 595, 3041-3051 (2017).

52 Kintzer, A. F., Sterling, H. J., Tang, II, Williams, E. R. \& Krantz, B. A. Anthrax toxin receptor drives protective antigen oligomerization and stabilizes the heptameric and octameric oligomer by a similar mechanism. PLoS One 5, e13888, doi:10.1371/journal.pone.0013888 (2010). 
53 McCowan, C. \& Garb, J. E. Recruitment and diversification of an ecdysozoan family of neuropeptide hormones for black widow spider venom expression. Gene 536, 366375, doi:10.1016/j.gene.2013.11.054 (2014).

672

54 Krasnoperov, V., Bittner, M. A., Holz, R. W., Chepurny, O. \& Petrenko, A. G.

673

674

675

676

677

678

679 Structural requirements for alpha-latrotoxin binding and alpha-latrotoxin-stimulated secretion. A study with calcium-independent receptor of alpha-latrotoxin (CIRL) deletion mutants. J Biol Chem 274, 3590-3596, doi:10.1074/jbc.274.6.3590 (1999).

55 Mosavi, L. K., Cammett, T. J., Desrosiers, D. C. \& Peng, Z. Y. The ankyrin repeat as molecular architecture for protein recognition. Protein Sci 13, 1435-1448, doi:10.1110/ps.03554604 (2004).

680

56 Li, J., Mahajan, A. \& Tsai, M. D. Ankyrin repeat: a unique motif mediating protein-

681 protein interactions. Biochemistry 45, 15168-15178, doi:10.1021/bi062188q (2006).

57 Li, J. D., Carroll, J. \& Ellar, D. J. Crystal structure of insecticidal delta-endotoxin

682

683

684

685

686

687 from Bacillus thuringiensis at 2.5 A resolution. Nature 353, 815-821, doi:10.1038/353815a0 (1991).

58 Xu, C., Wang, B. C., Yu, Z. \& Sun, M. Structural insights into Bacillus thuringiensis Cry, Cyt and parasporin toxins. Toxins (Basel) 6, 2732-2770, doi:10.3390/toxins6092732 (2014).

59 Gazit, E., La Rocca, P., Sansom, M. S. \& Shai, Y. The structure and organization within the membrane of the helices composing the pore-forming domain of Bacillus thuringiensis delta-endotoxin are consistent with an "umbrella-like" structure of the pore. Proc Natl Acad Sci U S A 95, 12289-12294, doi:10.1073/pnas.95.21.12289

692 (1998).

60 Ho, C. M. et al. Native structure of the RhopH complex, a key determinant of malaria parasite nutrient acquisition. bioRxiv, doi:10.1101/2021.01.10.425752 (2021).

693

694

61 Moldenhauer, H., Diaz-Franulic, I., Gonzalez-Nilo, F. \& Naranjo, D. Effective pore size and radius of capture for K(+) ions in K-channels. Sci Rep 6, 19893,

695

696

697 doi:10.1038/srep19893 (2016).

698

699 $\mathrm{Wu}$, J. et al. Structure of the voltage-gated calcium channel $\mathrm{Ca}(\mathrm{v}) 1.1$ at $3.6 \mathrm{~A}$ resolution. Nature 537, 191-196, doi:10.1038/nature19321 (2016).

700

63 Renart, M. L. et al. Effects of conducting and blocking ions on the structure and stability of the potassium channel KcsA. The Journal of biological chemistry $\mathbf{2 8 1}$, 29905-29915, doi:10.1074/jbc.M602636200 (2006).

703

64 Tang, L. et al. Structural basis for Ca2+ selectivity of a voltage-gated calcium channel. Nature 505, 56-61, doi:10.1038/nature12775 (2014). 
Figures

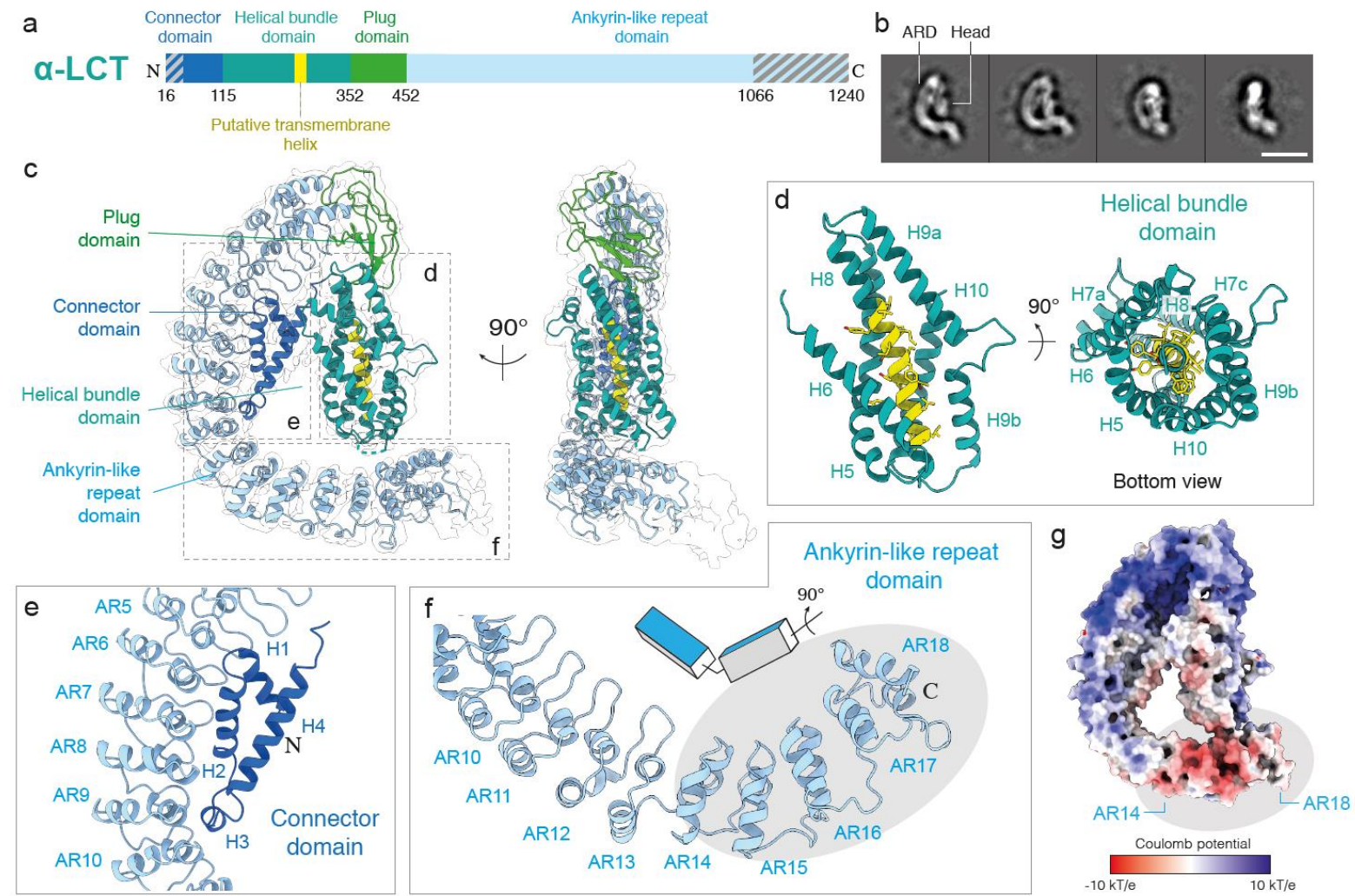

Figure 1. Structure of $\alpha$-LCT monomer. a, Domain organization of mature $\alpha$-LCT. Gray diagonal lines indicate regions not resolved in the cryoEM density. b, Representative reference free twodimensional class averages. Scale bar: $10 \mathrm{~nm}$. c, Side views of the $\alpha$-LCT monomer superposed with the EM map (transparent). Domains are depicted in the same colors as in a. d, Close-up view of the helical bundle domain. The front helix (H7) is not shown in the left image for clarity. e, Close-up view of the interface between CD (H1-3) and ARD (AR5-10). f, Close-up view of the ARD C-terminal tail. The gray ellipse indicates the last five ARs (AR14-18). Note the change in orientation $\mathbf{g}$, Electrostatic potential calculated in APBS. Red: -10 kT/e; Blue: +10 kT/e. PD:plug domain; HBD:helical bundle domain; CD: connector domain; ARD: ankyrin-like repeat domain 

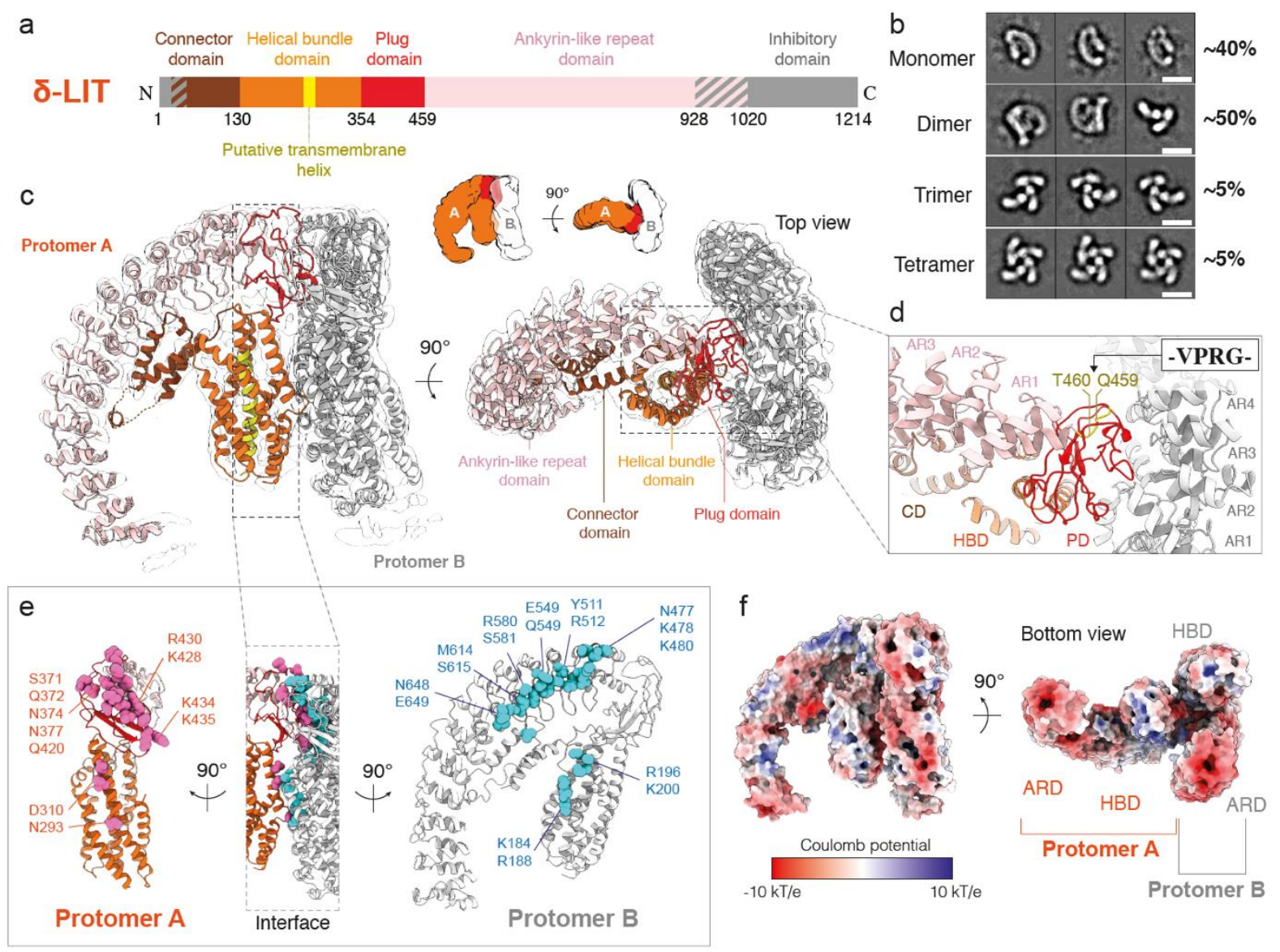

Figure 2. Structure of $\delta$-LIT dimer. a, Domain organization of full-length precursor $\delta$-LIT. Disordered regions in the cryoEM map are indicated in gray diagonal lines and boxes. b, Representative twodimensional class averages of each oligomeric state. Scale bar: $100 \AA$ A. c, Side and top view of the $\delta$-LIT dimer superposed with the EM map (transparent). Domains of protomer A are depicted in the same colors as in a; protomer B is colored in gray. $\mathbf{d}$, Close-up view of the PD-ARD dimerization interface. The position of the four amino acid insertion variant (VPRG) is indicated and colored in yellow. e, Side view of the dimerization interface. Protomers A and B are rotated $90^{\circ}$ to left and right, for better clarity. Polar and charged candidate residues $(<5 \AA$ to the opposite protomer) are shown as spheres and colored in cyan and pink. f, Electrostatic potential calculated in APBS. Red: $-10 \mathrm{kT} / \mathrm{e}$; Blue: +10 kT/e. PD:plug doman; HBD:helical bundle domain; CD: connector domain; ARD: ankyrin-like repeat domain 
a

$\alpha$-LCT

Compact
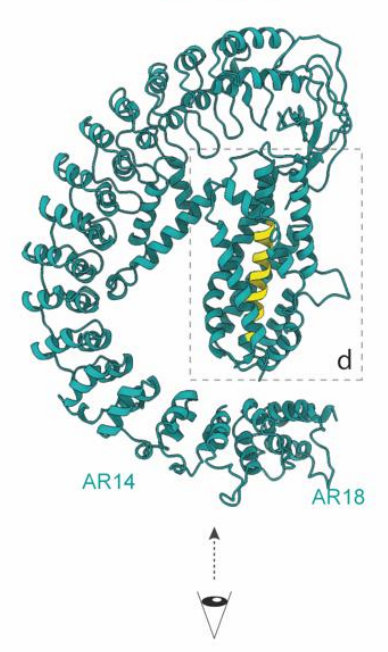

ס-LIT

Extended
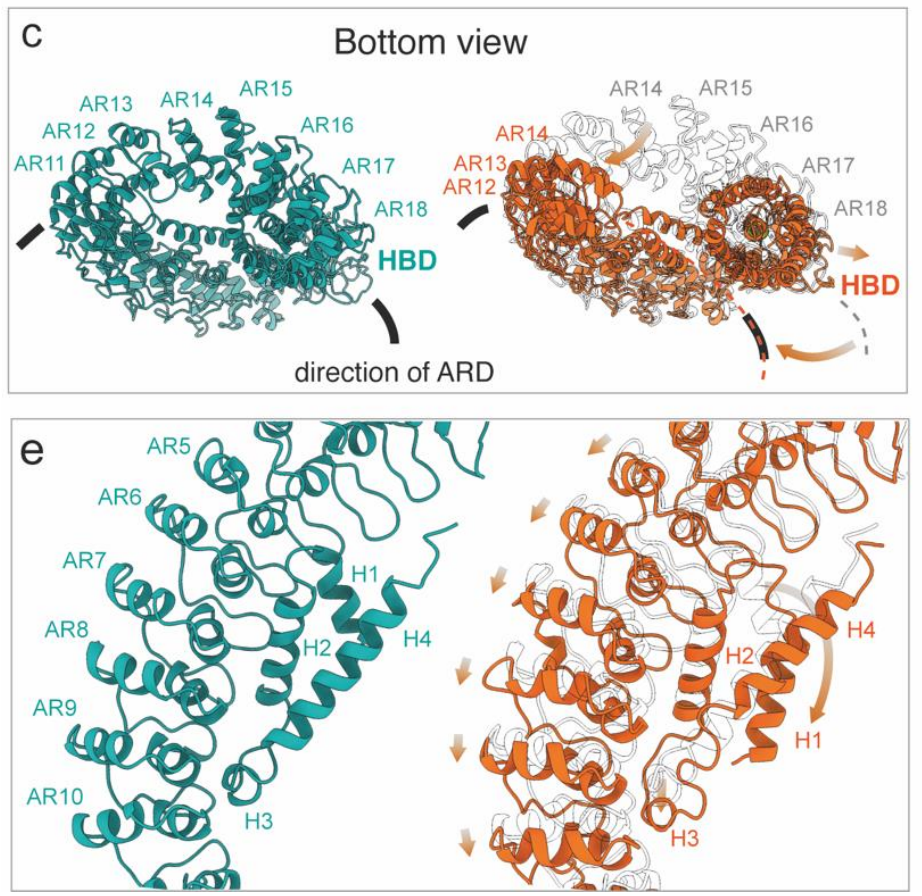

b

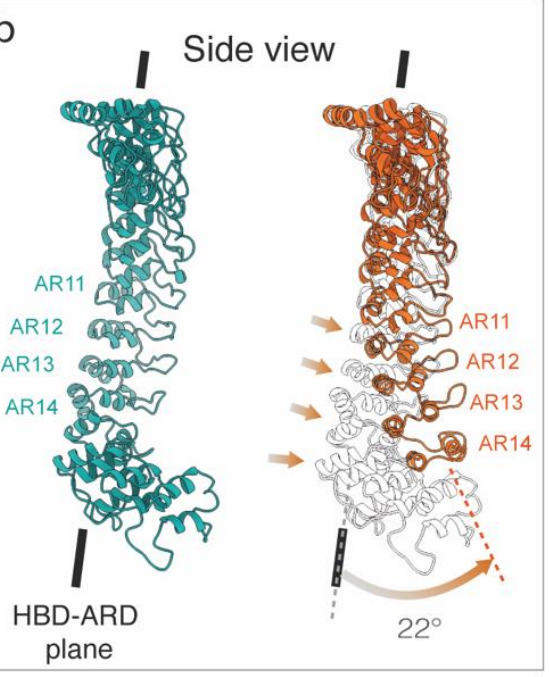

d

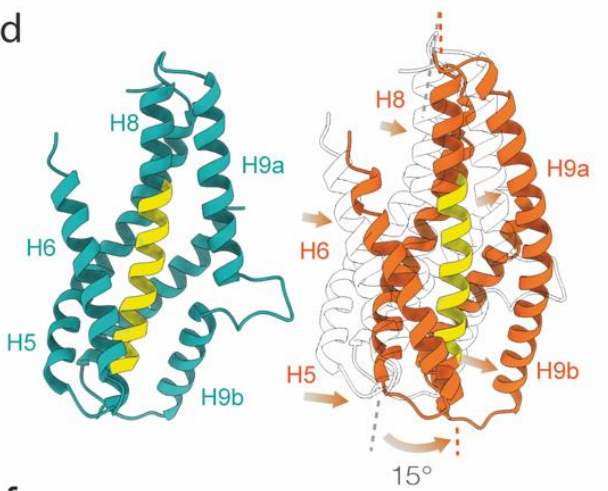

$f$

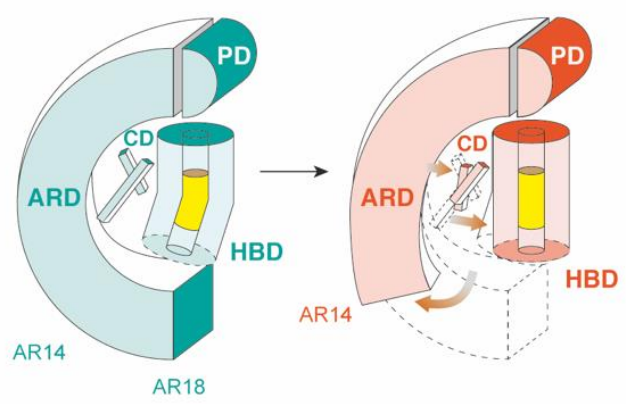

Figure 3. Conformational changes during dimerization. a Side-by-side comparison of $\alpha$-LCT (compact state, sea green) and d-LIT (extended state, extracted from the dimer, orange) superposed with $\alpha$-LCT (transparent). b, Front view of the ARD. Arrows indicate domain motion during dimerization $\mathbf{c}$, Bottom views of the compact and extended states. The bottom part of the cylindrical (HBD) is exposed in the extended state. d, Magnified view of the HBDs. The front helix (H7) is not shown for clarity. The H9a-H9b loop folds helically to complete H9 in the extended state. e, Magnified view of the interface between the connector- and the AR-domain $\mathbf{f}$, the schematic diagram illustrates the conformational change. PD:plug domain; HBD:helical bundle domain; CD: connector domain; ARD: ankyrin-like 
a

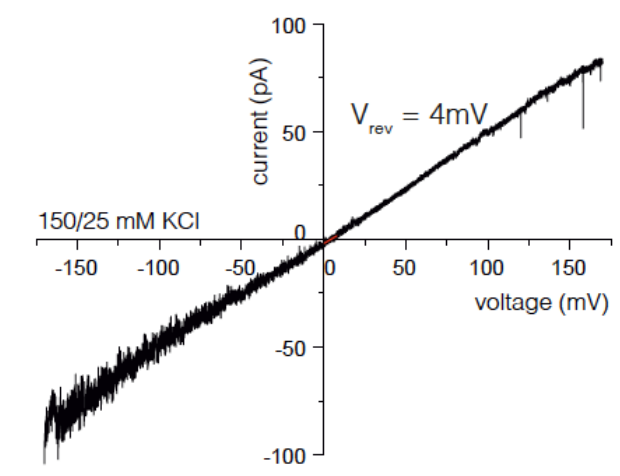

C

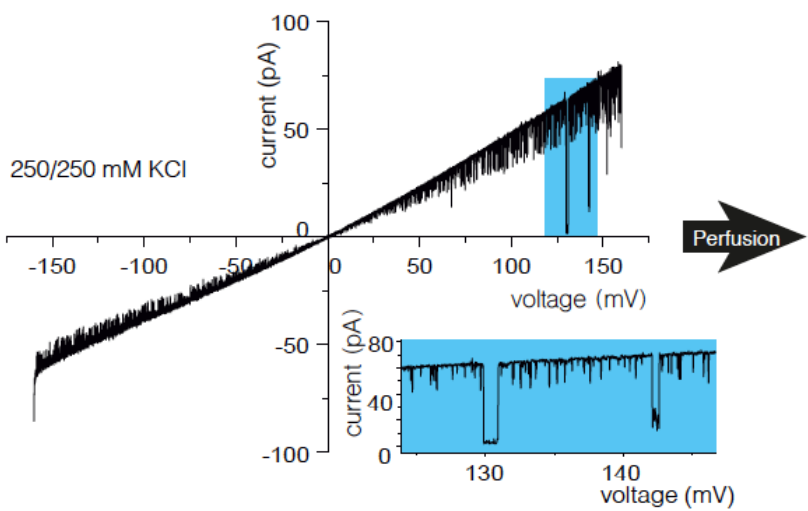

e

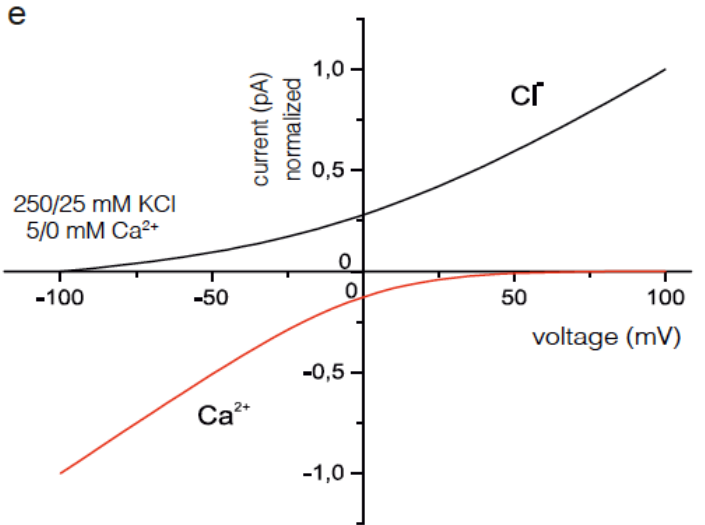

b

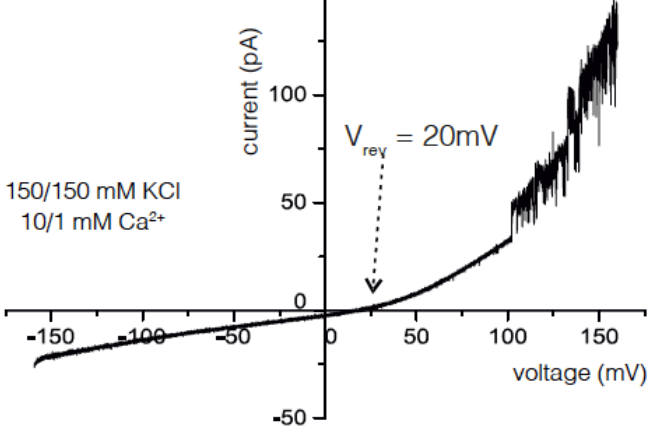

d
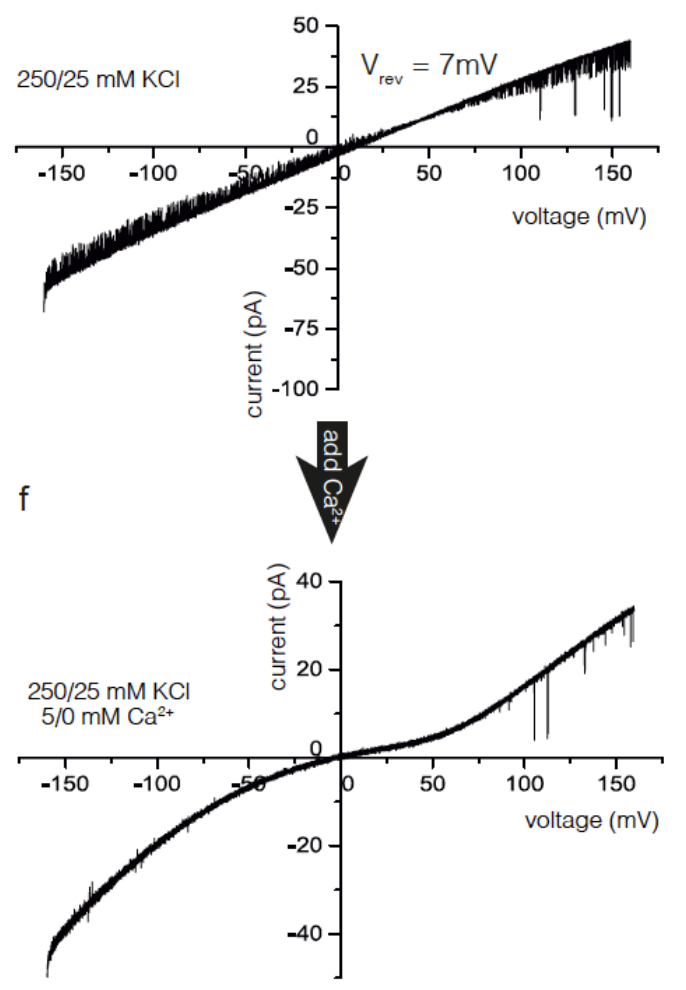

Figure 4. Calcium induced ion channel properties of reconstituted $\delta$-LIT variants within lipid bilayers.

Current-Voltage ramp recordings at different cis/trans buffer conditions. a, Precursor $\delta$-LIT shows a noisy linear current-voltage relation with reversal potential of $\mathrm{V}_{\text {rev }}=+4 \mathrm{mV}$, indicating a slight cation selective channel $\left(P_{K^{+}} / P_{C l^{-}}=1.25\right)$. b, With symmetric $\mathrm{KCl}$ and added asymmetric calcium the precursor $\delta$-LIT channel displays a $\mathrm{V}_{\text {rev }}=+20 \mathrm{mV}$, presenting now a highly $\mathrm{Ca}^{2+}$ selective channel $\left(P_{C a^{2+}} / P_{C l^{-}}=18\right)$, demonstrating a dramatic property change induced by the presence of $\mathrm{Ca}^{2+}$-ions. $\mathbf{c}$, The current-voltage relation of the mature $\delta$-LIT is again linear but with low current noise and frequent, defined gating from the open towards the closed state. (displayed in extension box). d, Next trans chamber perfusion within the same experiment. The $\mathrm{V}_{\text {rev }}=7 \mathrm{mV}$ value discloses cation selective properties $P\left({ }_{K^{+}} / P_{C l^{-}}=1.47\right)$ preserved within the mature variant. e, With asymmetric $\mathrm{KCl}$ and cis added calcium (no calcium addition trans) the precursor $\delta$-LIT channel displays an asymptotic sine currentvoltage relation with a zero-current crossing at $\mathrm{V}_{\text {rev }}=0 \mathrm{mV}$. Extrapolation of the tangent to zero net current yields $V_{\text {rev }}=\approx 40 \mathrm{mV}$ and a very high calcium selectivity of the mature $\delta$-LIT channel 
bioRxiv preprint doi: https://doi.org/10.1101/2021.04.19.440504; this version posted April 20, 2021. The copyright holder for this preprint (which was not certified by peer review) is the author/funder. All rights reserved. No reuse allowed without permission.

relative permeabilities from (e) and the experimental concentration of $\mathrm{Ca}^{2+}, \mathrm{K}^{+}$and $\mathrm{Cl}^{-}$ions in the cis and trans compartment (see Supplemental Information for Details).

758 


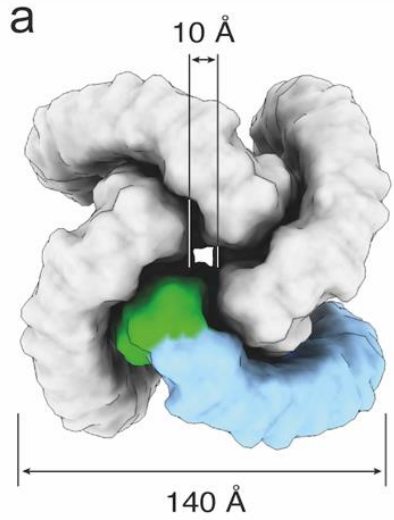

b

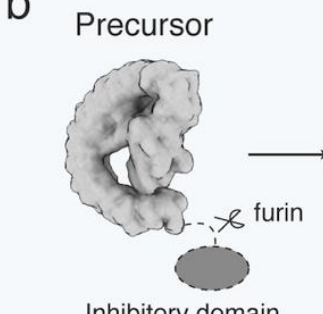

Inhibitory domain

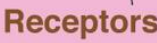

Receptors

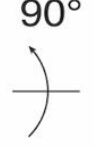

Compact

Figure 5. Latrotoxin tetramer assembly prior membrane insertion a, Simulated volume of a soluble $\boldsymbol{\delta}$-LIT insertion-competent tetramer. b, Model of latrotoxin action at the presynaptic membrane.
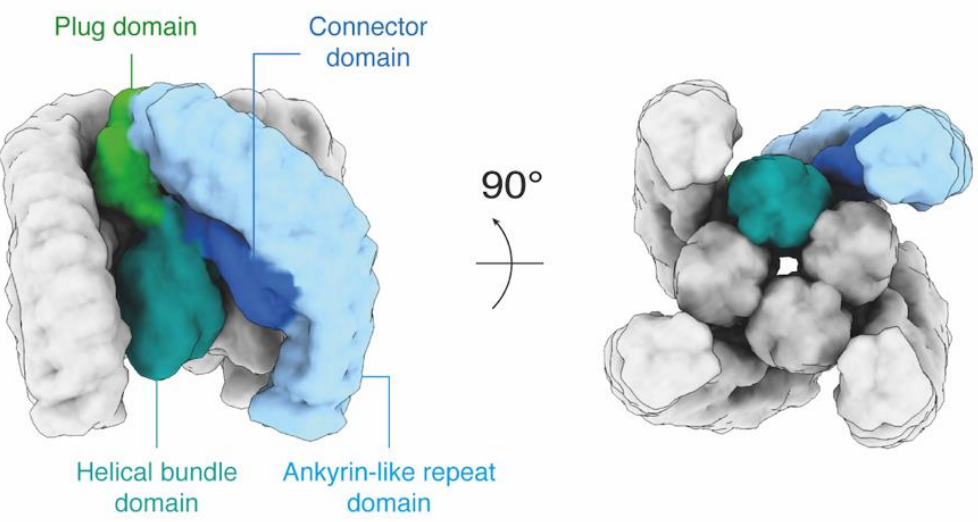

Extended

(intermediate)

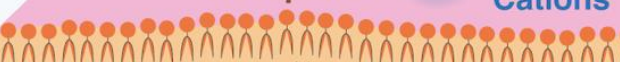

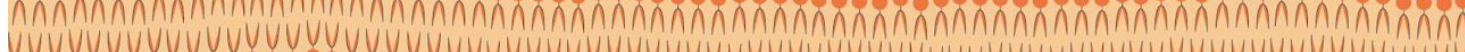

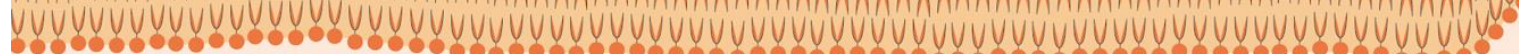

作 